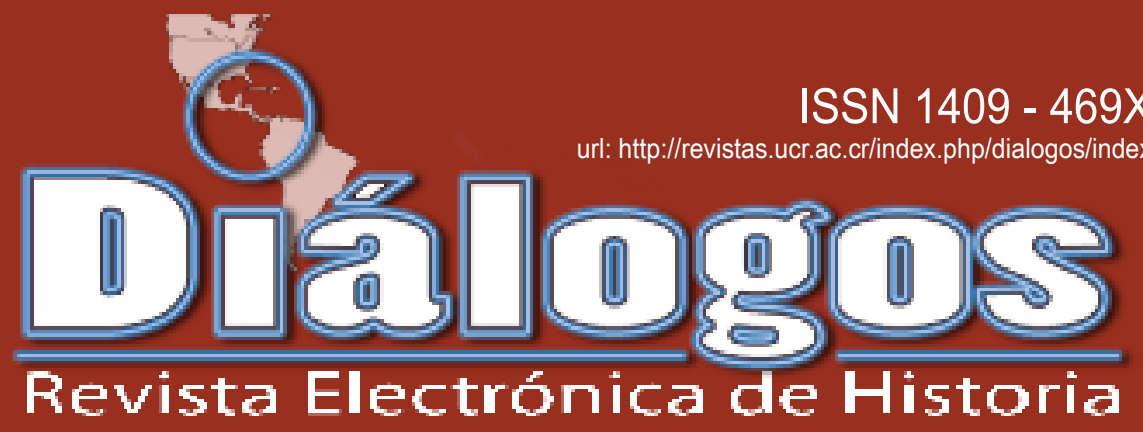

Escuela de Historia. Universidad de Costa Rica Volumen 14 especial en homenaje a Victor Hugo Acuña. Octubre 2013

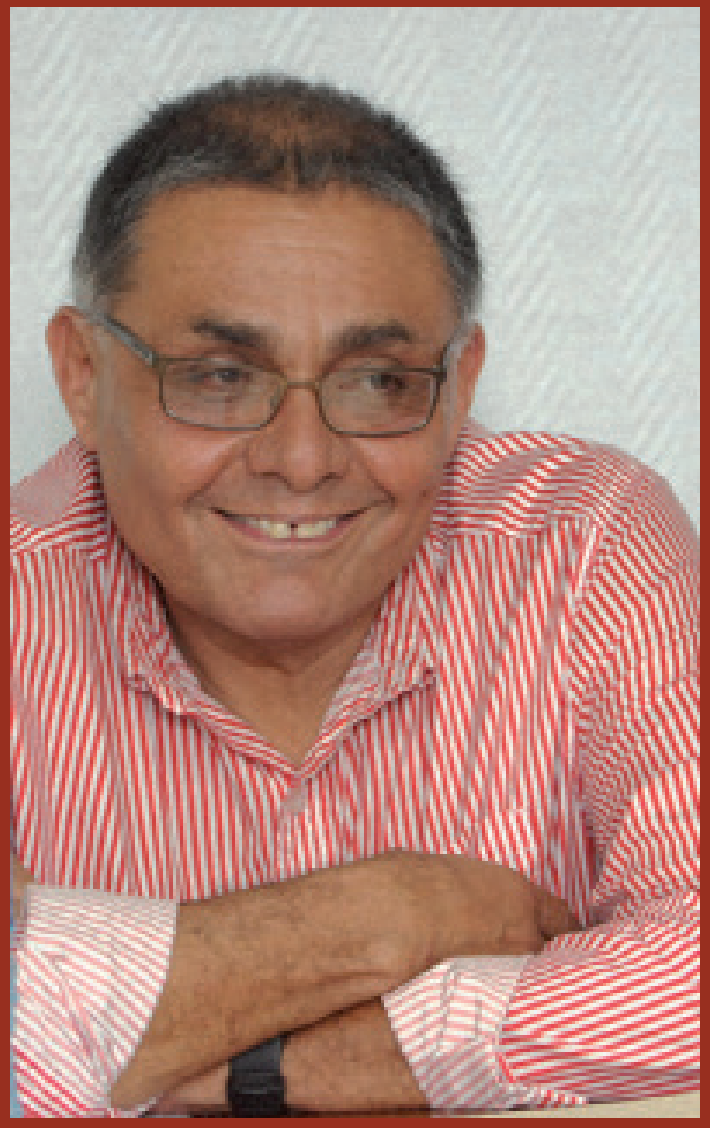

Director de la Revista: Dr. Juan José Marín Hernández juan.marinhernandez@ucr.ac.cr

Editor académico: Dr. Ronny Viales Hurtado - ronny.viales@ucr.ac.cr Editor técnico: M.Sc. Marcela Quirós G. - marcela.quiros@ucr.ac.cr 



\section{Miembros del Consejo Editorial:}

Dr. Juan José Marín Hernández, Catedrático. Director del Centro de Investigaciones Históricas de América Central. Universidad de Costa Rica. Costa Rica. juan. marin@ucr.ac.cr

Dr. Ronny Viales Hurtado. Catedrático. Historia Económica y Social. Universidad de Costa Rica. Director de la Escuela de Historia. Costa Rica. ronny. viales@ucr.ac.cr

Dr. David Díaz Arias: Catedrático. Historia Política, Director del posgrado de Historia y Docente de la Escuela de Historia, Universidad de Costa Rica, Costa Rica.david.diaz@ucr.ac.cr

MSc. Francisco Enríquez. Historia Social. Universidad de Costa Rica. Costa Rica. francisco.enriquez@ucr. ac.cr

Dra. Ana María Botey. Historia de los movimientos sociales. Universidad de Costa Rica. Costa Rica. abotey@gmail.com

\section{Miembros del Consejo Asesor Internacional:}

Dr. José Cal Montoya. Universidad de San Carlos de Guatemala. Guatemala. jecalm@correo.url.edu.gt

Dr. Juan Manuel Palacio. Universidad Nacional de San Martín. Argentina.jpalacio@unsam.edu.ar

Dr. Eduardo Rey. Universidad de Santiago de Compostela. España. ereyt@usc.es

Dr. Heriberto Cairo Carou. Departamento de Ciencia Política y de la Administración III Universidad Complutense de Madrid. España. hcairoca@cps.ucm.es

Dra. Rosa de la Fuente. Departamento de Ciencia Política y de la Administración III Universidad Complutense de Madrid. España. rdelafuente@cps. ucm.es

Dr. Javier Franzé. Departamento de Ciencia Política y de la Administración III Universidad Complutense de Madrid. España. javier.franze@cps.ucm.es

Dr. Jaime Preciado Coronado Departamento de Estudios Ibéricos y Latinoamericanos. Universidad de Guadalajara. México. japreco@hotmail.com
Dr. Gerónimo de Sierra. Vicerrector de la Universidade Federal da Integração Latino-Americana (UNILA) y Departamento de Sociología, Facultad de Ciencias Sociales de la Universidad de la República. Uruguay. geronimo@fcs.edu.uy

Dr. Antonio Palazuelos. Departamento de Ciencia Política y de la Administración III - Universidad Complutense de Madrid. España. palazuelosa@cps. ucm.es

Dr. Werner Mackenbach. Universidad Potsdam. Alemania. werner.mackenbach@uni-potsdam.de

Dr. Guillermo Castro. Ciudad del Saber Panamá. Panamá. gcastro@cdspanama.org

Dra. Natalia Milanesio. University of Houston. Estados Unidos.nmilane2@Central.UH.EDU

Dr. Ricardo González Leandri. Consejo Superior de Investigaciones Científicas - España. España. rgleandri@gmail.com

Dra. Mayra Espina. Centro de Estudios Psicológicos y Sociológicos, La Habana. Cuba.mjdcips@ceniai.inf.cu

Dra. Montserrat Llonch. Departamento de Economía e Historia Económica Universidad Autónoma de Barcelona. España.montserrat.llonch@uab.es

Dra. Estela Grassi. Universidad de Buenos Aires. Argentina. estelagrassi@gmail.com

Dra. Yolanda Blasco. Universidad de Barcelona. España. yolandablasco@ub.edu

Dr. Alfredo Falero. Departamento de Sociología. Universidad de la República. Uruguay. alfredof@adinet. com.uy

\section{Portada:}

Fotografía de Anel Kenjekeeva, Oficina de Divulgación Universidad de Costa Rica, publicada en http://www. ucr.ac.cr/noticias/2012/09/13/escuela-de-historia-rindehomenaje-a-victor-hugo-acuna.html

\section{Equipo Técnico Editorial:}

Editora Técnica: M.Sc. Marcela Quirós Garita.marcela. quiros@ucr.ac.cr

Diagramación: Cindy Chaves Uribe

Asistentes: Pablo Hurtado GranadosMaureen Méndez Montero 
"Diálogos Revista Electrónica de Historia" se publica desde octubre de 1999.

\section{Diálogos está en los siguientes repositorios:}

Dialnet

http://dialnet.unirioja.es/servlet/

revista?tipo_busqueda=CODIGO\&clave_revista $=3325$

Latindex

http://www.latindex.unam.mx/larga.php?opcion=1\&folio=12995;

\section{UCRindex}

http://www.revistas.ucr.ac.cr/

\section{Scielo}

http://www.scielo.cl/

\section{eRevistas}

http://www.erevistas.csic.es/

\section{REDALYC}

http://redalyc.uaemex.mx/src/inicio/FrmBusRevs2.jsp?iEdoRev=2\&cvepai=11;

\section{LANIC}

http://lanic.utexas.edu/la/ca/cr/indexesp.html

Repositorio de Revistas Universidad de Costa Rica http://www.latindex.ucr.ac.cr/

Directorio y recolector de recursos digitales del

Ministerio de Cultura de España

http://roai.mcu.es/es/inicio/inicio.cmd

DOAJ Directory of open access \& Hybrid journals

http://www.doaj.org/doaj?func=byTitle\&hybrid=1\&query=D

Biblioteca de Georgetown

http://library.georgetown.edu/newjour/d/msg02735.htm|

Asociación para el Fomento de los Estudios Históricos en Centroamérica

http://afehc. apinc.org/index.php?action=fi_aff\&id=1774

Universidad de Saskatchewan, Canadá

https://library.usask.ca/ejournals/view/1000000000397982

\section{Monografias}

http://www.monografias.com/Links/Historia/more12.shtml

\section{Hispanianova}

http://hispanianova.rediris.es/general/enlaces/hn0708.htm

Universidad del Norte, Colombia

http://www.uninorte.edu.co/publicaciones/memorias/enlaces.htm

Universidad Autónoma de Barcelona

http://seneca.uab.es/historia/hn0708.htm

Repositorio Invenia - Gestión del Conocimiento http://www.invenia.es/oai:dialnet.unirioja.es:ART0000086144

\section{Enlace Académico}

http://www.enlaceacademico.org/biblioteca/

revistas-en-formato-digital-centroamerica/

\section{Electronic Resources}

http://sunzi1.lib.hku.hk/ER/detail/hkul/3987318

Revistas académicas en texto completo http://web.prw.net/ vtorres/
Diálogos se anuncia en las siguientes instituciones y sitios académicos:

\section{Maestroteca}

http://www.maestroteca.com/detail/553/dialogos-revista-electronica-de-historia.htm

Biblioteca de Georgetown

http://library.georgetown.edu/newjour/d/msg02735.html

Asociación para el Fomento de los Estudios Históricos en Centroamérica

http://afehc. apinc.org/index.php?action=fi_aff\&id=1774

Universidad de Saskatchewan, Canadá

https://library.usask.ca/ejournals/view/1000000000397982

\section{Monografias}

http://www.monografias.com/Links/Historia/more12.shtml

\section{Hispanianova}

http://hispanianova.rediris.es/general/enlaces/hn0708.htm

Universidad del Norte, Colombia

http://www.uninorte.edu.co/publicaciones/memorias/enlaces.html

Universidad Autónoma de Barcelona

http://seneca.uab.es/historia/hn0708.htm

Repositorio Invenia - Gestión del Conocimiento

http://www.invenia.es/oai:dialnet.unirioja.es:ART0000086144

\section{Enlace Académico}

http://www.enlaceacademico.org/biblioteca

revistas-en-formato-digital-centroamerica/

\section{Electronic Resources}

http://sunzi1.lib.hku.hk/ER/detail/hkul/3987318

Revistas académicas en texto completo

http://web.prw.net/ vtorres/

La revista electrónica Diálogos es financiada por Vicerrectoría de Investigación de la Universidad de Costa Rica

\section{Citado en: Scielo -}

Dialnet - eRevistas

- UCRindex - Latindex -

REDALYC - DOJAC - Directorio

y recolector de recursos

digitales del Ministerio de

Cultura de España

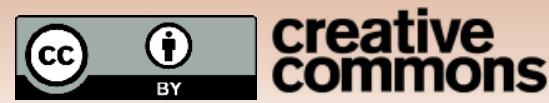





\title{
SOBRE UN DÍA LEJANO Y EL PASO DEL TIEMPO
}

ON AN DISTANT DAY AND HOW TIME GOES BY

Víctor H. Acuña Ortega

\author{
Palabras claves \\ Pasado, Universidad de Costa Rica.
}

Keywords

Past, University of Costa Rica.

Fecha de recepción: 17 de mayo, 2013 - Fecha de aceptación: 8 de agosto 2013

\section{Resumen}

El autor realiza un análisis en diferentes niveles, sobre el día en que ingresó a la Universidad de Costa Rica.

\section{Abstract}

The author analyzes the day he enroled the University of Costa Rica. 



\section{SOBRE UN DÍA LEJANO Y EL PASO DEL TIEMPO}

Víctor H. Acuña Ortega

A la memoria de mi padre Víctor Manuel Acuña García.

Tras la triple emoción de sorpresa, contento y agradecimiento al recibir la noticia por parte de David Díaz de la organización de esta actividad, me surgió la pregunta de lo que yo debía decir en tal ocasión, pregunta que se transformó al cabo de algunas semanas en su formulación negativa: lo que no debía decir en esta ceremonia. Supongo que hay distintas posturas que en estas circunstancias se deben evitar, pero al menos yo pensé en dos: la primera sería la que llamaría la del veterano de guerra que viene a discurrir sobre las cicatrices en su cuerpo y en su memoria que dan testimonio de sus glorias de antaño; y la segunda, que identificaría como la postura del predicador, es decir, la de quien viene con el ceño fruncido a dar lecciones a las generaciones actuales sobre lo que se debe o no se debe hacer. En efecto, sentí que a mí no me cuadraba la postura del veterano, del ancien combattant, como dirían los franceses, que no pierde la ocasión en cualquier reunión social para evocar los viejos buenos tiempos, los cuales, por supuesto eran mejores a los actuales y, en donde al final, si tiene a su lado a otros camaradas, termina por concluir, víctima de una de esas pérfidas ilusiones de la astuta memoria, que los mariscos de esa época eran muy superiores a los de hoy porque aquellos sí eran potentes y eficaces en cuanto a sus virtudes como afrodisíacos; chanza que tomo prestada de un viejo amigo aquí presente. Pero aún más me parecía insostenible la postura del predicador moralista que tiene el don mágico de saber de antemano y desde siempre donde se encuentran los malos y donde se sitúan los buenos, con lo cual se siente en el derecho de repartir los respectivos premios y castigos entre ambos grupos. Esta actitud me repugna y me asusta porque, como sabemos, es la de los que encienden hogueras y levantan patíbulos, y quizás también porque en algunos momentos se coló en mis ardores de juventud, cuando creía que era posible conocer y conducir las llamadas leyes de la historia. (Otro amigo me recordó en días recientes, con gracia y simpatía, ese viejo pecado). Debo agregar que soy muy sensible a esa postura porque es discernible entre historiadores de viejo cuño, de ayer y de hoy, que confunden en sus obras elogio o condena con explicación. En suma, con la conciencia de que debía evitar esas dos trampas, la de quien se ancla en un pasado fantasmal y la de aquel que pretende oficiar como tribuno de la historia, al cabo de varios meses, al fin encontré lo que consideré era pertinente para esta tarde, ni homilía, ni reminiscencia, sino una especie de simulación, un ejercicio, breve y modesto, de práctica delante de ustedes de cómo, según mi experiencia, se elabora la historia como saber empírico. 
Para los efectos de esta charla, tenía que encontrar un objeto delimitado y delimitable, el cual permitiera ser sometido a la prueba de dos interrogantes: el primero, es si se puede articular un relato a partir de una serie de informaciones necesariamente fragmentarias, es decir, si es posible un emplotment, como dirían los narrativistas y narratólogos, con un puñado de objetos dispersos, encontrados al azar del tiempo, y el segundo, si es posible asomarse al tiempo del mundo y al tiempo de un mundo por la mirilla, tronera o claraboya de un breve momento y obtener con esa mirada una comprensión esencial de ambos. En otras palabras, tenía dos preguntas: ¿es realista pretender contar una historia con dos o tres vestigios de experiencias pretéritas?; y ies factible adivinar o identificar las claves del mundo y de ese mundo en el cual esa historia habría acontecido? En sentido estricto, las dos preguntas son puramente retóricas porque, como sabemos, de ese modo trabajan los historiadores: construyendo narraciones históricas a partir de objetos encontrados, que los historiadores llamamos fuentes, arbitraria y fortuitamente transportados en el tiempo, y tratando de encontrar la lógica y los fundamentos de estructuración y movimiento de los universos en donde las historias acontecen. Pero, como también lo sabemos, los historiadores suelen desbordarse de placer y de excitación cuando encuentran en abundancia lo que llaman sus fuentes. De todos modos, no se puede negar que, en principio, con pocos rastros es poquito lo que se puede decir. En fin, consciente de los riesgos y las dificultades que conlleva, este es el contrato que me he propuesto y que les propongo esta tarde: hablar de un instante fugaz y tratar de encontrar su significado o quizás, con menos ambición, algunos de sus significados.

Ese momento, quizás ustedes ya lo intuyen, es un día, un solo día de mi vida, día compartido por muchos de ustedes que son mis contemporáneos, pero no tan lejano en el tiempo para aquellos de ustedes de cohortes más recientes. Debo advertir que se trata de veinticuatro horas más bien anodinas, como suelen ser la mayoría de los días de cada año, en los cuales los ritmos de la naturaleza y de la vida social se suceden en repetición y en los cuales los llamados acontecimientos apenas son pliegues con poca consecuencia sobre la superficie lisa de la duración. Ciertamente que existen los días de grandes acontecimientos, aquellos que representan un punto de giro en la historia, como ese de agosto de 1914 con el cual, dirían algunos historiadores, terminó el largo siglo XIX y comenzó el siglo XX, o como aquel otro de noviembre de 1989 cuando, se nos dice, acabó el corto y mortífero siglo XX y se inició esta marcha incierta por el nuevo milenio. Pero el día del que me voy a ocupar no se recuerda por nada en especial y es previo a grandes acontecimientos de mis años universitarios; pienso en la muerte del Ché Guevara, la desintegración de los Beatles, Mayo 68, el descenso de Armstrong en la luna o aún los sucesos de ALCOA, símbolo del encuentro con la historia con mayúscula de la generación a la cual pertenezco. En fin, es un día del año 1967, de marzo de 1967: el día en que ingresé a estudiar a la Universidad de Costa Rica. 
Confiado en mi memoria, la cual me recordaba que por aquellos años el curso lectivo en nuestra universidad empezaba el primer lunes de marzo, me fui a consultar el archivo del diario La Nación, disponible por Internet. Revisé el número de ese día, pero me pareció que había algo raro o que en todo caso en el diario no se consignaba nada sobre ese evento de la vida universitaria. Sentí una especie de sospecha e incertidumbre respecto a lo que tenía por seguro y decidí revisar, en forma rápida, las ediciones de los días siguientes y precedentes y aún así no puede resolver el pequeño enigma. Consulté con un amigo aquí presente, funcionario y estudiante universitario en ese entonces, quien basado en su recuerdo me confirmó que el inicio de clases era el primer lunes de marzo. No obstante, como persistía mi sensación de incertidumbre decidí visitar el Archivo Universitario para ver si ellos conservaban los calendarios del curso lectivo. Me dijeron que no y me remitieron al archivo del Consejo Universitario. Aquí, una funcionaria amable, servicial y competente, se encargó de localizar las actas respectivas, ya que la fijación del calendario universitario era potestad de ese organismo. Tales actas me permitieron establecer que para el año 1967 el Consejo Universitario había determinado adelantar el inicio del curso lectivo al día primero de marzo, a pesar del desacuerdo de la representación estudiantil.

Lamentablemente, la duda no se disipó porque luego consulté el número de La Nación del primero de marzo y allí me encontré el siguiente texto de la famosa Columna, a cargo del periodista Manuel Formoso Peña, denominada, como recordarán algunos entre los aquí presentes, en los círculos de izquierda y de la progresía del Partido Liberación Nacional, "La Calumnia". Así, en dicha sección de opinión de ese diario de aquel día encontré el texto siguiente:

\begin{abstract}
"El lunes se iniciarán las clases en la Universidad de Costa Rica y este año, según informes dados a la prensa, la matrícula alcanzará la respetabilísima suma de seis mil seiscientos alumnos. Ya están alistando, los de segundos y terceros años, las máquinas de pelar, con ánimo de desquitarse de la pelada que les suministraron a ellos, Y, por los datos que han trascendido, van a usar la número cero que deja el pelado a $\mathrm{CO}-\mathrm{CO}$, y no a GO-GO, que es con una melenita muy YE-YE.” (“La Columna”, La Nación, 1 de marzo de 1967, p.4.)
\end{abstract}

Como se puede ver, la tarea de identificar las fuentes históricas pertinentes para el ejercicio que me había propuesto se había tornado complicada y prolongada. No me ayudó la circunstancia de que inicialmente había previsto leer los periódicos, todos los del día seleccionado, en su versión en papel en la hemeroteca de la Biblioteca Nacional. Allí acudí, pero se me informó que no era posible consultar los periódicos antiguos, por obras que se realizan en la sección donde se encuentran depositados. La funcionaria que me atendió, muy amablemente, me dijo que podía volver en el mes de noviembre cuando esa sección volvería a estar abierta al público. Yo le dije que necesitaba hacer la consulta ya; entonces, siempre amable- 
mente, me remitió a que probara suerte en la biblioteca de la Asamblea Legislativa, lo cual deseché ad portas, o que fuese a la UCR, sugerencia por la cual me decidí. En fin, en la UCR descubrí que se encontraban disponibles los diarios La Prensa Libre y La República en forma microfilmada, pero que había que leerlos en negativo y en una máquina que posiblemente fue adquirida, no mucho tiempo después de aquel día cuyos periódicos pretendía localizar. En suma, decidí contentarme con la lectura del diario La Nación por Internet, desde la comodidad de mi casa; menester en el que se me atravesó, para alargar mis penas, la pana reciente de este servicio en la red del ICE, de la cual soy usuario.

Al fin, gracias a las actas del Consejo Universitario y gracias a dos avisos publicados en La Nación los días 26 y 27 de febrero pude determinar que en 1967 el curso lectivo se inició de la manera siguiente: el primero de marzo empezaron sus labores docentes toda las escuelas de la universidad, salvo las escuelas de Ingeniería, Ciencias Económicas y Sociales, Derecho, y Ciencias y Letras que comenzaron su curso lectivo el 2 de marzo. Así, como Humanidades, la puerta de entrada de los estudiantes de primer ingreso, era parte de Ciencias y Letras, pude al fin decir que mi primer día como estudiante de la Universidad de Costa Rica fue el jueves 2 de marzo de 1967. Cualquier duda sobre este asunto se disipó cuando leí en la sección de Sociales de este diario del viernes 3 de marzo que ese día en la noche se realizaría, en el Centro de Recreación Estudiantil, el tradicional baile de bienvenida a los nuevos estudiantes de la UCR, organizado por la Federación de Estudiantes Universitarios, llamado el "Baile de los Cocos". En suma, tras un inesperado y aleccionador recorrido por lo que suele ser el trabajo de investigación histórica pude establecer mi objeto de estudio y sus respectivas fuentes.

Cuando por primera vez formule la idea de este experimento, me dije: voy a analizar lo que pasó en Costa Rica y en el mundo el día en que yo ingresé a la UCR. Un tiempo después, me percaté de mi ingenuidad epistemológica, como dirían los que se dan de posmodernos, ya que efectivamente lo que en esos universos aconteció aquel día es infinito y en algún sentido incognoscible. En realidad, dado que me basaba en la prensa diaria como fuente, de lo que efectivamente me iba a ocupar era de la actualidad de aquel día, es decir, de lo que fue considerado actual para ese día por periodistas, redacción y dirección de este periódico, actualidad, por lo demás, referida a eventos del día anterior. Así, en sentido estricto, me iba a ocupar de lo que había sido acontecimiento el día previo a mi ingreso a la UCR, según este principio de producción de la actualidad. Ciertamente, que hubo otras actualidades no solo en otras partes del mundo ( hubiese podido, por ejemplo, consultar el New York Times de ese mismo día disponible en Internet, mediante el respectivo pago), sino también en Costa Rica, en el sentido de que los otros diarios o el semanario comunista Libertad no necesariamente informaron sobre los mismos temas y eventos, ni con los mismos énfasis. 
Además, de esta cuestión de cómo es construida la percepción de la realidad social había de por medio otro problema más de fondo. En efecto, la actualidad no es la misma para todo el mundo no solo en términos discursivos, sino también en términos reales ya que según la expresión de un filósofo alemán, existe la simultaneidad de lo no simultáneo o como la llama otro autor la contemporaneidad de lo no contemporáneo, es decir, no todos los que vivimos en el mismo tiempo astronómico compartimos el mismo tiempo histórico y social. En la Costa Rica de aquellos años había grupos de seres humanos y lugares en donde la actualidad de la que hablaba La Nación, no se situaba en el mismo plano temporal de su tiempo vivido. En suma, se trataba de la actualidad definida desde la capital de Costa Rica y para determinados grupos sociales del país. Esa actualidad y esa contemporaneidad son las que voy a presentar ahora en este ejercicio.

Me voy a permitir entonces transcribir los titulares del diario La Nación (72 páginas, treinta céntimos el número suelto y seis colones la suscripción mensual), del 2 de marzo de 1967, día en que a eso de las siete de la mañana apenas al bajar del bus al frente de la iglesia de San Pedro fui atacado por unos sujetos armados de tijeras, quienes sin contemplación me dejaron la melena en jirones.

Titulares principales:

Informa al país la Cámara de Comercio de su labor de mediación: la citada cámara ha mediado entre el gobierno, la oposición liberacionista y los sectores empresariales para encontrar una solución a la crisis fiscal y monetaria que atraviesa el país. Su principal propuesta es el establecimiento del impuesto de ventas.

Con interés y respeto estudiamos propuesta de la Cámara de Comercio: Trejos.

Libre exportación de ganado vacuno.

Solución inmediata al problema de la leche propone el gobierno.

Pie de Foto: Funcionarios del gobierno de Vietnam del Sur examinan el primer donativo del pueblo de Honduras llegado a esta capital y consistente en alimentos y medicinas. El donativo llegó a bordo de un avión militar de la fuerza aérea hondureña.

Los otros titulares:

Clausurado el tercer curso de instructores del Movimiento Nacional de Juventudes.

Tendrá trascendental importancia: se refiere a una conferencia de los países miembros del Sistema Interamericano que se realizará entre el 12 y el 14 de abril próximos.

Beneficios por \$27 millones en las futuras siembras de banano en Río Frío.

Diputados al margen de la ley: informa que 11 diputados no han pagado los derechos de importación de sus vehículos, cuyos números de placa se consignan.

Guanacaste reaccionó contra la entrega de las cañerías al SNAA.

Malestar por recurso de inconstitucionalidad entre los diputados de la oposición: se refiere a que el Ejecutivo rehúsa girar a los diputados las partidas específicas. 
Apoya la Cámara de Industrias la patriótica labor desplegada por la Cámara de Comercio.

Las restricciones bancarias obligarán al abandono de cultivos expresó la Cámara de Agricultura ayer al Presidente Trejos. Esta información trata sobre el financiamiento por parte de la banca nacionalizada de la cosecha cafetalera.

Reconocimiento de la Cámara de Industrias a La Nación por su desinteresada campaña a favor de la industria nacional.

2.500 .000 colones anuales para impulsar la reforma penitenciaria.

En la sección de opinión del periódico cabe señalar "La Columna", "Comentarios", es decir, el editorial, y la columna diaria de ANFE. También los lectores opinan por medio de la sección "Cartas a La Columna" y hay una contribución sobre la candidatura de Gonzalo Facio Segreda a la Secretaría General de la OEA, de Marciano Campos Bolaños, célebre en aquellos años, apodado "Marciano Campos Pagados", quien vivía en una cruzada perpetua contra el comunismo, tanto el internacional como el criollo y que, en efecto, publicaba en la prensa con bastante frecuencia campos pagados, lo que hacía preguntarse a más de uno de donde obtenía el dinero para pagar sus campos. Hay otros artículos de opinión, uno de Claudio Gutierrez, quien aún no era rector de nuestra universidad, en defensa de la planificación familiar, adversada, como pueden imaginar, por la jerarquía de la Iglesia Católica costarricense, y otro de Cristián Rodríguez, quien llegó a ser uno de los colaboradores estrella de la sección de opinión de este diario conocida como la "Página Quince", creada por Guido Fernández años después, sobre la influencia en su generación cuando se inició a la vida consciente, hecho que sitúa hacia 1915, del libro Degeneración, publicado en 1892, de Max Nordau, un escritor judío sionista nacido en Budapest, crítico del arte decadente y de la modernidad, muy en consonancia con la atmósfera de declive del imperio austro-húngaro a fines del siglo XIX; autor, agrego, para mí desconocido.

Debo advertir, para subrayar el carácter retrospectivo del análisis histórico, que un contemporáneo de esta actualidad pudo haber hecho un análisis de coyuntura, como suelen hacerlo algunos colegas sociólogos o politólogos o un balance crítico como los que hacen algunos periodistas que se posicionan como analistas competentes de la actualidad. En este sentido, esto es posible para quien es contemporáneo de los eventos, pero no para quien se ocupa de ellos mucho tiempo después, para lo cual debe comenzar por establecerlos porque en principio los desconoce. Obviamente, la ventaja del historiador en relación con el analista de la coyuntura o el comentarista de la actualidad es que conoce el desenlace de los acontecimientos y el lugar que ocupan en una cadena temporal de largo plazo. En fin, no se debe creer que el ejercicio que me propongo sea muy original porque en el último medio siglo, dentro de las distintas corrientes de la historia social y cultural, se pueden encontrar 
obras que han intentado reconstruir la historia de un solo día o la biografía de un individuo anónimo y ordinario, seleccionado al azar en el fichero de un archivo.

Hecha esta advertencia sobre mi lugar en el tiempo respecto de aquella actualidad y sus consecuencias sobre la condición de mi análisis, cuales podían ser las preguntas que yo podía formularme frente a esta serie de titulares. Desde que me surgió la idea lo que me interesaba identificar era la diferencia entre aquel presente y el nuestro, es decir, si había una radical alteridad entre aquel marzo de 1967 y este septiembre de 2012; pero también quería palpar en qué sentido aquel tiempo compartía una cercanía con el actual. La pregunta puede parecer en una primera impresión bastante ingenua puesto, que como sabemos, la actualidad es por definición efímera y pasajera y, en ese sentido, cada actualidad es fugaz, única y distinta de cualquier otra como bien lo expresa la canción de Héctor Lavoe "Periódico de ayer", ese que nadie quiere ya leer. De este modo, la pregunta más bien remite a una problemática clásica de los historiadores: la cuestión de la continuidad y la discontinuidad de los procesos históricos. Así, debo confesar que al ver este diario de ese día lo que me sorprendió fue su familiaridad o parentesco con el tiempo presente. Sobre todo, esta impresión me surgió al ver la publicidad, muy abundante por cierto, en la cual se evidenciaba que la sociedad costarricense de aquellos años ya estaba totalmente enrumbada hacia el consumo de masas. Ciertamente, que no había malls, pero sí Más por Menos, hoy devenido Walmart. Tampoco voy a negar el fenómeno ya señalado de la contemporaneidad de lo contemporáneo o para emplear un término marxista, de aquellos tiempos, hoy en desuso, el desarrollo desigual y combinado; pero lo cierto que la impresión que me dejó la lectura de ese diario fue la de que la sociedad costarricense era una sociedad realmente moderna, en términos de lo que podríamos llamar las estructuras de la vida cotidiana.

Contribuyó a la percepción de familiaridad con aquel pasado de casi medio siglo la circunstancia de la continuidad del marco político institucional y también la presencia central del Estado en Costa Rica. Un Estado que se proponía a sí mismo como el principal agente y gestor de la modernidad; misión que podía enfrentar resistencia en algunos lugares como Guanacaste en donde las municipalidades y quizás también los vecinos se oponían al traspaso de sus servicios de agua potable al SNAA. Si pensamos en lo que ocurrió en Centroamérica y en otros países latinoamericanos en las décadas de 1970 y 1980, es posible afirmar que la estabilidad del régimen político costarricense después de la guerra civil de 1948 es un factor poderoso para que se experimente esa sensación de continuidad entre aquel presente y el nuestro. También, aunque resulte un poco anecdótico, señalarlo, hay una continuidad en relación con algunos problemas estructurales del Estado costarricense, es decir, sus crónicos problemas fiscales y sus recurrentes relaciones incestuosas con la Iglesia costarricense.

No obstante, por supuesto que estamos en otra época. Para empezar, la matriz 
de las relaciones a nivel global era el conflicto de la Guerra Fría, como nos lo recuerda la foto de primera página y como, de una manera ridícula y estrafalaria para nosotros, podemos constatarlo en una carta a "La Columna", titulada, posiblemente por la redacción del periódico, "Muchos rusos en programas universitarios", en la cual su autor, Pedro Corrales de oficio constructor, se queja del "rusismo" de los programas musicales de la Radio Universitaria. Veamos lo que dice:

\footnotetext{
"Hoy, por ejemplo, por un solo Beethoven nos sirven dos Procofiev (sic), dos Korsakoff, además de un Stravinsky, y aparte de los que invariablemente van apareciendo en los llamados CONCIERTOS PARA COMPLACER SOLICITUDES (?), [con mayúsculas y acompañado de un signo de pregunta] dos o tres cantantes rusos que le salen a uno por sorpresa en cada programa."

"Cartas a la Columna", p. 2.
}

Mi comentario sale sobrando, aunque conviene agregar que en la actualidad internacional de ese día, además de la guerra de Vietnam, también está presente la revolución cultural china en curso, la cual es presentada como otra forma de barbarie del comunismo, la revolución cubana y la lucha guerrillera en Guatemala.

También es evidente que aquella es otra etapa de la historia económica de Costa Rica, centrada en el desarrollo industrial, acompañado de la integración económica centroamericana, y la diversificación de las exportaciones, como es el caso de la ganadería, y la renovación de la producción bananera en el Caribe, aunque la producción cafetalera sigue siendo clave. La lectura de La Nación de aquel día muestra claramente su afinidad ideológica con el sector industrial, de la cual da testimonio su apoyo a la campaña Compre y use lo que Costa Rica produce, mediante la inserción de un cintillo con esa frase, reproducido a lo largo de las distintas páginas del periódico. Debo confesar que había olvidado que este diario tuvo un pasado ideológico proteccionista; postura que se expresa de manera firme y contundente en el editorial de ese día, el cual se pronuncia en favor de que la inminente construcción de la carretera El Coco-San Ramón le sea adjudicada a empresas costarricenses. (p. 4) Ciertamente que el nacionalismo económico no es precisamente algo que forme parte del kit ideológico actual del Grupo Nación. También me llamó la atención la visibilidad en la esfera pública de las cámaras empresariales, aunque quizás esto sea algo propio también del tiempo presente. En todo caso, la Cámara de Comercio y la Cámara de Industrias ya no tienen el protagonismo de aquel entonces.

La información sobre el Movimiento Nacional de Juventudes y el ya citado fragmento de "La Columna" sobre ese mundo de Go-Gos, Ye-Yes y Co-Cos muestran que en aquellos años se empezaba a manifestar un actor social y cultural del que luego se hablaría muchísimo, en los años justo inmediatos, es decir, los jóvenes y los estudiantes. En la Costa Rica de aquel momento los jóvenes seguían aún por los cauces de sus mayores, como quizás podamos inferirlo de la actividad del "Baile 
de los cocos". Aún no era posible adivinar lo que iba a pasar con ellos posteriormente, pero sí es claro que comenzaba a manifestarse en Costa Rica ese fenómeno que fue tan típico de la década de 1960 y de la siguiente: los movimientos juveniles y estudiantiles, como retadores de las estructuras de dominación del capitalismo, en lo cual al final fueron poco eficaces, y como impugnadores de la moral y los estilos de vida burgueses, en lo cual nuestra generación puede decir que sí hizo una contribución importante. En este sentido, para un lector como yo de esta época resulta realmente muy exótica la llamada sección de Sociales, prácticamente desaparecida de nuestra prensa hace ya bastante tiempo. A través de ella se adivinan los convencionalismos, los formalismos, el conservatismo y el persistente aldeanismo en el modo de vida que caracterizaban a la sociedad costarricense de aquel entonces.

Dicho esto, vuelvo a insistir en la percepción que deja el diario de esta sociedad como una sociedad moderna. Modernos también somos nosotros e incluso podemos decir que somos practicantes, sin querer queriendo, de la condición posmoderna y es aquí en donde se dibuja una diferencia radical entre aquella sociedad y la nuestra. Las personas de aquel día o una buena parte de ellas, en todo caso, no solo se adherían a lo que consideraban moderno, sino que, sobre todo, creían en el progreso y estaban convencidas de que el futuro por delante era prometedor. En ese sentido, abrazaban el régimen de historicidad, término de un historiador francés, del futuropasado, en el cual el porvenir era brillante y el pasado ya no era ni referente, ni valor a respetar. Quizás sea sintomático al respecto, los avisos publicados por la CCSS en esos días ofreciendo plazas para médicos en distintos lugares del país. Aquí radica quizás la diferencia más profunda entre aquel presente y el nuestro en la medida en que la ideología del progreso ha entrado en declive y la mirada que prevalece en amplios sectores del mundo occidental y en múltiples grupos en Costa Rica es el pesimismo y el temor al porvenir. El régimen de historicidad en el que vivimos hoy, denominado presentismo, se ha quedado sin pasado y sin futuro y tiene como única opción un presente siempre en fuga, arrastrado por las utopías tecnológicas llevadas a la esfera del consumo personal y de la vida íntima.

Al final de este recorrido selectivo por el diario La Nación del 2 de marzo de 1967 talvez podríamos sostener que mi apuesta de mirar la marcha del mundo y de nuestro pequeño mundo por la estrecha mirilla que nos ofrece la actualidad de un solo día ha sido exitosa en la medida en que con las informaciones así obtenidas pude esbozar esquemáticamente algunas líneas básicas, del espíritu o, para emplear un término más neutral, del talante de aquella época o de aquellos años. También podría sostener que con esas fuentes que decidí utilizar he sido capaz de sugerir un relato aceptablemente coherente sobre la Costa Rica y el mundo de ese día, con base en las nociones de modernización, Guerra Fría, desarrollo industrial, diversificación económica, Estado, régimen político, etc. Así, en ese caos aparente era posible encontrar una lógica entre acontecimientos dispersos, como lo hubiese 
podido hacer alguien contemporáneo que hubiese intentado un análisis de coyuntura, aunque sin nuestra ventaja de conocer los desenlaces de esta historia. No obstante, aquí hay una trampa porque el análisis que he presentado no se ha basado exclusivamente en la información de este diario de ese día, ya que, como dije, además revisé otros números, anteriores y posteriores, de dicha publicación, aunque aquí haya hecho caso omiso de ellos y hayan quedado solo como un conocimiento implícito. Mi análisis también se ha sustentado en la memoria, en mis recuerdos, en la medida en que fui contemporáneo de aquellos acontecimientos. Una memoria selectiva y fragmentaria, como cualquier memoria, porque ni siquiera me acordaba del día exacto en que ingresé a la Universidad de Costa Rica y mucho menos de los titulares de La Nación de ese día. No obstante, está claro que mi análisis no se ha basado únicamente, ni quizás principalmente, en las fuentes de que dispuse, sino en el conocimiento de su contexto, producto tanto de lo vivido como de lo leído y estudiado, ya que yo enseñé cursos sobre la historia de Costa Rica del siglo XX, en distintas ocasiones.

En este punto de mi ejercicio de simulación de investigación histórica es donde quisiera insistir en un principio metodológico elemental de la disciplina que se olvida cuando se cree que historia es sinónimo de erudición, es decir, los datos no significan nada si no se ponen a la luz de un contexto, tanto analítico, como conceptual; un vestigio o rastro que carece de contexto es mudo, no dice nada o prácticamente nada. Pero el reconocimiento de la necesidad del contexto, no implica que sea prescindible el texto, lo cual parece evidente. Pero con esto quiero en específico recordar las trampas de la memoria; no solo porque nos crea a veces la ilusión de hacernos creer que sabemos, como que nos predispone a perder una mirada distanciada en relación con el pasado. De esta manera, mi testimonio y mis recuerdos no han reemplazado como lo he demostrado lo que está escrito en ese diario del 2 de marzo de 1967.

Para terminar, quisiera ocuparme del otro segmento del título de esta charla: el paso del tiempo. Ha quedado manifiesto que aquel mundo es distinto del nuestro, aunque ambos guarden continuidades entre sí. La Costa Rica actual no se ha alejado definitivamente de aquella, porque quizás muchos de los estábamos entre los vivos en aquel entonces seguimos, para nuestra dicha y tal vez para la de otros, estando vivos hoy. También como lo señalan quienes estudian la historia de la actual fase del mundo, la llamada globalización, es posible que sus orígenes inmediatos remonten a aquellos años. Ahora bien, es importante recordar que los de aquel entonces nunca supimos como sería el mundo de hoy, por más capacidad de prognosis de algunos. Este presente es el producto del paso del tiempo, pero de un tiempo lleno de desenlaces contingentes, nunca totalmente predeterminados. Yo mismo jamás hubiera podido imaginar este día y esta circunstancia porque, para empezar, aquel primer día que ingresé a la universidad fue para estudiar economía, no historia. Además, 
mi vida ha sido una cadena de encuentros y de resultados inesperados. En todos ellos, sé perfectamente, soy deudor de muchas personas. Siempre he sabido que siempre he estado en el lugar donde he estado en este tránsito permanente gracias a muchas personas, unas aquí presentes, otras lejanas y otras para siempre ausentes. Me encantaría nombrarlas a todas, pero aquí solo me puedo contentar con pronunciar los nombres de dos ausentes: Carmen Ortega Canales, mi madre, y Humberto Salas González, amigo entrañable.

Tres Ríos, septiembre de 2012

\section{ACERCA EL AUTOR}

Víctor H. Acuña Ortega: Doctor en Historia por la École des Hautes Études en Sciences Sociales Université de Paris-Sorbonne. Es profesor emérito de la Universidad de Costa Rica. E-mail: vhacuna@gmail.com 
